\title{
Studien über Metallcyanate
}

\author{
ERIK S ÖDERB Ä CK
}

\author{
Chemisches Institut der Universität Uppsala, Schweden
}

\begin{abstract}
Es wurden vorher unbekannte Cyanate von Cadmium und von einund zweiwertigem Kupfer synthetisiert und von beiden letzteren Doppelcyanate mit Alkalimetallen.

Ein katalytischer Effekt der Cuprocyanate bei der Einführung der Isocyanatgruppe in einen Benzolkern nach Sandmeyer wurde entdeckt und als Beispiel die Synthese von o-Tolylisocyanat durchgeführt.

Neue Methoden für die Darstellung von Mercuricyanat sind ausgearbeitet worden; diese Substanz wurde näher studiert und Ähnlichkeiten mit dem Mercurichlorid nachgewiesen. Eine neue bequeme Methode für die Darstellung von Bariumcyanat ist angegeben und die Löslichkeit des Salzes in Wasser bestimmt worden.

Die Leitfähigkeitbestimmungen und einige der Analysen sind im hiesigen Centralanalytischen Laboratorium, Vorstand Dr. F. Nydahl, ausgeführt worden. Für diese sowie für Debye-Scherrer-Aufnahmen durch Herrn Privatdozent Dr. I. Lindqvist bin ich sehr zu Dank verpflichtet.
\end{abstract}

$D^{\mathrm{i}}$ ie Metallcyanate sind im Verhältnis zu den Metallrhodaniden recht wenig untersucht worden, und viele einfache Cyanate von gewöhnlichen Metallen sind noch unbekannt. Der Grund dafür ist hauptsächlich die relative Schwäche und Unbeständigkeit der Cyansäure in wässriger und alkoholischer Lösung. Die Säure wird in wässriger Lösung rasch zu Ammoniak und Kohlensäure hydrolysiert, in alkoholischer sind Urethane und Allophansäureester die Reaktionsprodukte.

Das Cyanation wird häufig als ein Analogon der Hypohalogenitionen, z.B. des Hypochloritions betrachtet, weil es durch Hydrolyse des Dicyans in alkalischer Lösung nebst Cyanidion gebildet wird. Diese Übereinstimmung ist aber ausschliesslich formal, da keine Übereinstimmung in Eigenschaften besteht; die Cyanate haben z.B. keine oxydierende Wirkungen. Das Cyanation zeigt vielmehr gewisse Ähnlichkeiten mit den Halogenidionen, namentlich mit dem Chloridion, und diese sind besonders bei gewissen Schwermetallcyanaten hervorstechend. So ist z.B. schon längst ein Kaliumchloroplatinatcyanat $\mathrm{K}_{2} \mathrm{Pt}(\mathrm{OCN}) \mathrm{Cl}_{5} \mathrm{H}_{2} \mathrm{O}$ bekannt ${ }^{1}$.

Das Cyanation besitzt indessen eine mehr ausgeprägte Neigung zur Bildung von schwerlöslichen Doppelcyanaten als das Chloridion zu Bildung von Doppel- 
chloriden, was bei der Darstellung einfacher Metallcyanate manchmal Schwierigkeiten macht *. Es ist mir gelungen die einfachen Cyanate von Cadmium und Kupfer darzustellen und Doppelsalze von ein- und zweiwertigem Kupfer mit Alkalicyanaten. Ferner sind neue Methoden zur Darstellung von Mercuricyanat aufgefunden worden, ein Salz das grosse Ähnlichkeiten mit dem Chlorid aufweist.

\section{Cadmium cyan at}

Versetzt man eine nicht zu verdünnte Wasserlösung von einem normal ionisierten Cadmiumsalz mit Kaliumcyanat, kristallisiert das $\mathrm{Salz} \mathrm{KCd}(\mathrm{OCN})_{3}$ rasch aus ${ }^{3}$. Die Bereitung des einfachen Cyanats als Hydrat bietet indessen keine grössere Schwierigkeiten; es kann, wie ich gefunden habe, entweder durch Umsatz von Cadmiumsulfat mit Bariumcyanat in verdünnter wässriger Lösung und Konzentrieren der Lösung durch Vakuumdestillation bis zur eintreten der Kristallisation oder durch Reaktion von Cadmiumjodid in Acetonlösung mit der genau äquivalenten Menge Silbercyanat und Auslagen des Gemenges von $\mathrm{AgJ}$ und $\mathrm{Cd}(\mathrm{OCN})_{2}$ mit Wasser gewonnen werden. Das aus Wasser kristallisierte Cyanat ist immer etwas von basischem Salz verunreinigt und löst sich deshalb nicht klar in Wasser, es kann aber leicht durch Waschen mit verdünnter Essigsäure ** gereinigt werden und ist dann analysenrein. Die Zusammensetzung entspricht der Formel $2 \mathrm{Cd}(\mathrm{OCN})_{2} \cdot 5 \mathrm{H}_{2} \mathrm{O}$, der Wassergehalt ist also derselbe als für Cadmiumchlorid ***. Das Salz ist in Methanol und Äthanol ziemlich löslich.

Die Löslichkeiten in Wasser von Cadmiumsalzen der zusammengesetzten Halogene $(\mathrm{OCN})_{2},(\mathrm{SCN})_{2}$ und $(\mathrm{SeCN})_{2}$ sind nicht besonders gross. Nach von mir ausgeführten Messungen sind sie bei $20^{\circ}$ für $2 \mathrm{Cd}(\mathrm{OCN})_{2} \cdot 5 \mathrm{H}_{2} \mathrm{O} 9,1 \times 10^{-2}$, für $\mathrm{Cd}(\mathrm{SCN})_{2} 4,8 \times 10^{-2}$ und für $\mathrm{Cd}(\mathrm{SeCN})_{2} 2,3 \times 10^{-2} \mathrm{M}$. Die beiden letzten Salze kristallisieren anhydrisch und können aus konzentrierten $\mathrm{Cd}\left(\mathrm{NO}_{3}\right)_{2}$ Lösungen durch KSCN bezw. KSeCN gefällt werden. Der Grund für die relative Schwerlöslichkeit dieser Salze in Vergleich mit $\mathrm{CdCl}_{2}, \mathrm{CdBr}_{2}$ und $\mathrm{CdJ}_{2}$ dürfte in Autokomplexbildung zu suchen sein.

\section{Mercuricyanat}

Von Birckenbach und Kolb ${ }^{6}$ sind eine Reihe von Doppelcyanaten von Quecksilber(II) hergestellt und studiert worden, von welchen durch Ramananalyse einige als O-Cyanate andere als N-Cyanate zu formulieren seien. Die Verhältnisse sind indessen sehr verwickelt, und die Strukturen können kaum als endgültig geklärt betrachtet werden. Von denselben Autoren ist auch das einfache Mercuricyanat $\mathrm{Hg}(\mathrm{OCN})_{2}$ dargestellt worden durch Umsetzung von $\mathrm{HgCl}_{2}$ mit $\mathrm{AgOCN}$ in Methanol und Verdampfen des Lösungsmittels. Die Struktur soll nach Ramanaufnahmen der Ket-Imidformel entsprechen, was

\footnotetext{
* Bei den mit den Cyanaten isomeren Fulminaten ist diese Tendenz noch stärker ausgeprägt.

** Über die relative Stärke von Essigsäure und Cyansäure, siehe weiter unten.

*** Das bei Zimmertemperatur aus wässriger Lösung kristallisierte Cadmiumchlorid ist nach Untersuchungen von Cohen ${ }^{4}$ und Dietz ${ }^{5}$ das $\mathrm{Hydrat} \mathrm{2CdCl}_{2} \cdot 5 \mathrm{H}_{2} \mathrm{O}$. Diese Tatsache ist seit Ende des vorigen Jahrhunderts bekannt. Nichts destoweniger wird das käufliche Chlorid von chemischen Fabriken als Dihydrat formuliert.
} 
an und für sich nicht unwahrscheinlich ist. Methanol dürfte indessen kein geeignetes Solvenz für Mercuricyanat sein wegen mangelnder Stabilität des Salzes in diesem Lösungsmittel. Um es rein zu erhalten muss es sehr schnell aus der Lösung isoliert werden. Wie ich gefunden habe, ist es viel besser Äther als Solvenz zu verwenden, in welchem die Reaktion glatt verläuft. Es wird zwar von Birckenbach und Kolb behauptet, dass $\mathrm{Hg}(\mathrm{OCN})_{2}$ in Äther wenig löslich sei; nach meinen eigenen Messungen ist die Löslichkeit bei $20^{\circ} 1,3 \mathrm{~g}$ per $100 \mathrm{ml}$, eine Löslichkeit die ausreicht um das Salz mit Äther gut extrahieren zu können. Bei Abdestillieren des Äthers kristallisiert $\mathrm{Hg}(\mathrm{OCN})_{2}$ in analysenreiner Form aus. Es dürfte dies die beste Darstellungsmethode sein.

Mercuricyanat kann ferner, wie ich gefunden habe, durch Neutralisation von gelbem $\mathrm{HgO}$ mit Cyansäure in Äther gewonnen werden. Die Reaktion muss aber einen möglichst rapiden Verlauf haben, weil sonst durch eine sekundäre Reaktion zwischen $\mathrm{HgO}$ und $\mathrm{Hg}(\mathrm{OCN})_{2}$ letzteres in eine weisse schwerlösliche Substanz verwandelt wird, die nicht durch HOCN in normales Cyanat umgewandelt wird. Das $\mathrm{HgO}$ muss deshalb höchstreaktiv, in der Kälte dargestellt sein. Die Cyansäure lässt sich sehr bequem durch Umsatz von AgOCN mit $\mathrm{HCl}$ in trocknem Äther darstellen *. Die Isolierung des $\mathrm{Hg}(\mathrm{OCN})_{2}$ geschieht wie oben beschrieben, und die Ausbeuten sind befriedigend. Keine Unterschiede zwischen dem aus $\mathrm{HgO}$ und dem aus $\mathrm{HgCl}_{2}$ in Methanol synthetisierten $\mathrm{Hg}(\mathrm{OCN})_{2}$ konnten bei Debye-Scherreraufnahmen im hiesigen Institut durch Lindqvist beobachtet werden.

Das Mercuricyanat lässt sich auch nach einer dritten Methode gewinnen und zwar durch Erhitzen von Mercurocyanat $\mathrm{Hg}_{2}(\mathrm{OCN})_{2}$ mit Wasser, wobei jenes in Quecksilber und $\mathrm{Hg}(\mathrm{OCN})_{2}$ zerfällt. Es stellen sich Gleichgewichte ein, die sich mit der Temperatur stark verschieben; bei Zimmertemperatur wird die Reaktion quantitativ rückgängig. Durch Erneuerung der Wasserphase kann die Dissoziation von $\mathrm{Hg}_{2}(\mathrm{OCN})_{2}$ leicht vollständig gemacht werden. Durch möglichst rasches Einengen der abgekühlten Wasserextrakte mittelst Vakuumdestillation wird das Cyanat fraktioniert auskristallisiert und durch Umlösen in Äther gereinigt. Es konnten in dieser Weise Ausbeuten von etwa $80 \%$ erreicht werden.

Das Mercuricyanat kristallisiert aus Äther in prismatischen Kristallen, die sich in Licht etwas gräulich färben und deshalb im Dunkeln aufzubewahren sind. Die Löslichkeit in Äther nimmt mit dem Wassergehalt des Äthers zu, was auch für Quecksilberchlorid gilt. Während in trocknem Äther die Löslichkeit bei $20^{\circ} 1,3 \mathrm{~g} / 100 \mathrm{ml}$ beträgt, steigt sie für einen Äther, dessen Wassergehalt $0,1 \mathrm{M}$ ist $\mathrm{zu} 1,8 \mathrm{~g}$ und für $0,25 \mathrm{M} \mathrm{zu} \mathrm{2,7} \mathrm{g}$. Diese Löslichkeiten wurden durch Wägen des Verdampfungsrückstandes eines abgemessenen Volumens gesättigter Lösung bestimmt. $\mathrm{Hg}(\mathrm{OCN})_{2}$ ist wie $\mathrm{HgCl}_{2}$ leicht löslich in einer Menge von organischen Solvenzien, in Methanol und Äthanol, in aliphatischen Nitrilen wie Acetonitril, in den verschiedensten aliphatischen Ketonen, wie Aceton, Methyläthylketon und $C y c l o h e x a n o n$. Es ist ferner beträchtlich löslich in Wasser. $100 \mathrm{ml}$ lösen bei $20^{\circ} 7,5 \mathrm{~g} \mathrm{Hg}(\mathrm{OCN})_{2} * *$ und $5,7 \mathrm{~g} \mathrm{HgCl}_{2}$. Bei $0^{\circ}$

* Ganz analog lässt sich eine ätherische Rhodanwasserstofflösung durch Umsatz von AgSCN mit $\mathrm{HCl}$ bereiten.

** Die Löslichkeit von $\mathrm{Hg}(\mathrm{ONC})_{2}$ in Wasser ist bei $18^{\circ} 0,74 \mathrm{~g}$ in $100 \mathrm{ml}$. 
sinkt die Löslichkeit für das Cyanat zu 3,7 $\mathrm{g}$ und für das Chlorid zu 3,6 g. Eben wie $\mathrm{HgCl}_{2}$ ist $\mathrm{Hg}(\mathrm{OCN})_{2}$ in Wasser anomal wenig ionisiert. Alkalicarbonate erzeugen keinen Niederschlag von $\mathrm{HgO}$, wohl aber Alkalihydroxyde. $\mathrm{AgNO}_{3}$ fällt nicht; aus Lösungen von $\mathrm{HgCl}_{2}$ wird bekanntlich $\mathrm{AgCl}$ partiell gefällt. Es ist dazu zu bemerken, dass das Löslichkeitsprodukt von $\mathrm{AgCl}, 2 \times 10^{-10}$ nicht unwesentlich kleiner ist als dasjenige von $\mathrm{AgOCN}, \sim 10^{-7}$. Dass $\mathrm{Hg}(\mathrm{OCN})_{2}$ anomal wenig ionisiert ist, geht aus der Tabelle 1 hervor, wo die molekularen Leitfähigkeiten $\Lambda$ bei $25^{\circ}$ in reciprok. Ohm für 4 verschiedene Verdünnungen angeführt sind. Die letzte Kolumne enthält die Leitfähigkeiten für $\mathrm{HgCl}_{2}$ bei denselben Verdünnungen nach Ley ?. Die Zeiten $Z$ sind von dem Augenblick, wo das Salz mit dem Lösungsmittel in Berührung kam, gerechnet.

Es geht aus der Tabelle hervor, dass die Leitfähigkeit von $\mathrm{Hg}(\mathrm{OCN})_{2}$ von derselben Grössenordnung wie diejenige von $\mathrm{HgCl}_{2}$ ist *. Sie steigt mit der Zeit, offenbar durch eine Reaktion des Salzes mit Wasser. Die wässrigen Lösungen von $\mathrm{Hg}(\mathrm{OCN})_{2}$ sind nämlich nicht stabil, sondern setzen allmählich gelblich gefärbte Niederschläge ab unter gleichzeitiger Entwicklung von $\mathrm{CO}_{2}$. Über die Art dieser Reaktion kann gegenwärtig nichts bestimmtes gesagt werden. Auch in wasserhaltigem Äther wird $\mathrm{Hg}(\mathrm{OCN})_{2}$ allmählich zersetzt.

Die Mercurisalze der elementaren Halogene sowie diejenige der zusammengesetzten $(\mathrm{SCN})_{2}$ und $(\mathrm{SeCN})_{2}$ werden bei Zimmertemperatur in Wasser oder organischen Solvenzien leicht und quantitativ durch Quecksilber zu den entsprechenden Mercurosalzen reduziert und derselben Reaktion unterliegt auch das Mercuricyanat. Es wurden Versuche in Wasser und Äther angestellt. Das Abschliessen der Reaktion konnte leicht durch Ausbleiben der Ausfällung von $\mathrm{HgO}$ bei Zusatz von Alkalihydroxyd, im Falle Äther auch durch das Verschwinden von Eindampfungsrückstand festgestellt werden, da Mercurocyanat in Äther sehr schwerlöslich ist. Die Identität des Reaktionsproduktes mit $\mathrm{Hg}_{2}(\mathrm{OCN})_{2}$ konnte dadurch gezeigt werden, dass beim Aufkochen mit Wasser $\mathrm{Hg}(\mathrm{OCN})_{2}$ ausgelöst wurde und wie oben beschrieben, isoliert werden konnte.

Das Mercuricyanat gibt genau wie $\mathrm{HgCl}_{2}$ die Edersche Reaktion d.h. es wird in wässriger Lösung bei Gegenwart von $\left(\mathrm{NH}_{4}\right)_{2} \mathrm{C}_{2} \mathrm{O}_{4}$ durch Belichtung zu $\mathrm{Hg}_{2}(\mathrm{OCN})_{2}$ reduziert unter gleichzeitiger Bildung von $\mathrm{CO}_{2}$

$2 \mathrm{Hg}(\mathrm{OCN})_{2}+\left(\mathrm{NH}_{4}\right)_{2} \mathrm{C}_{2} \mathrm{O}_{4}+h v \longrightarrow \mathrm{Hg}_{2}(\mathrm{OCN})_{2}+2 \mathrm{NH}_{4} \mathrm{OCN}+2 \mathrm{CO}_{2}$

Bei einem derartigen Versuch wurden $16,6 \mathrm{~g} \mathrm{Hg}(\mathrm{OCN})_{2}$ in einer Lösung von $17 \mathrm{~g}\left(\mathrm{NH}_{4}\right) \mathrm{C}_{2} \mathrm{O}_{4}$ in $400 \mathrm{ml}$ Wasser aufgelöst mit einer Quarzquecksilberlampe mehrere Tage belichtet. Es wurden dabei $12,7 \mathrm{~g} \mathrm{Hg}_{2}(\mathrm{OCN})_{2}$ ausgefällt $=89 \%$. Das Präparat war durch kleine Mengen von ausreduziertem $\mathrm{Hg}$ etwas gräulich gefärbt.

Von Interesse ist, dass die Doppelverbindungen $\mathrm{Hlg} \cdot \mathrm{HgOCN}(\mathrm{Hlg}=\mathrm{Cl}$, Br oder J) eben wie die entsprechenden Rhodanide und Selenocyanide Phototropie zeigen. Die Empfindlichkeit steigt von $\mathrm{Cl} \mathrm{zu} \mathrm{J} \mathrm{an}{ }^{8}$.

Wie schon erwähnt, ist Mercuricyanat in Methanol, Äthanol und aliphatischen Ketonen leichtlöslich. Wenn eine Lösung von anhydrischem Cupri-

* Das mit Mercuricyanate isomere Fulminat hat eine Leitfähigkeit die nicht merkbar grösser als diejenige von Wasser ist.

Acta Chem. Scand. 11 (1957) No. 10 
Tabelle 1.

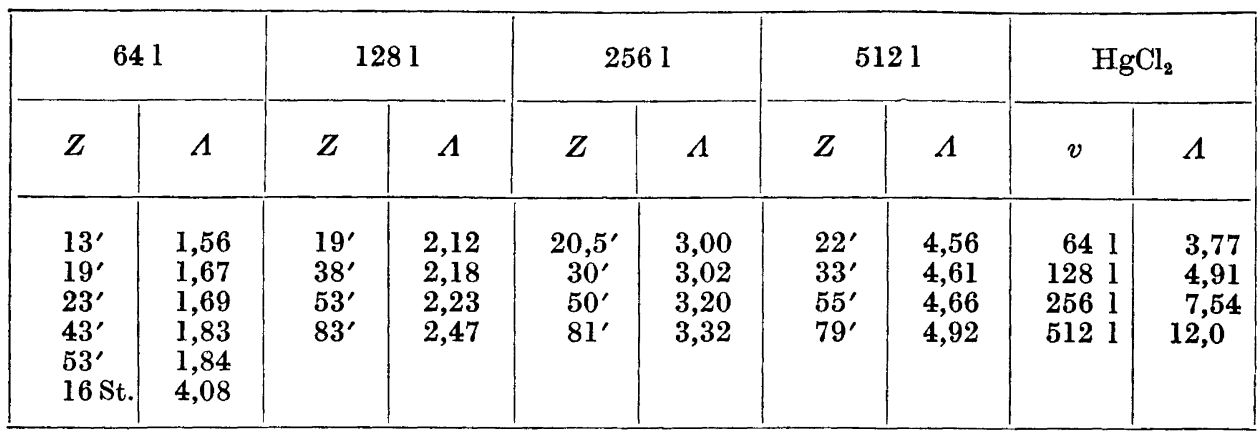

bromid in Methanol oder Äthanol mit einer äquivalenten Menge von $\mathrm{Hg}(\mathrm{OCN})_{2}$ in demselben Solvenz versetzt wird, so schlägt die braune Farbe der $\mathrm{CuBr}_{\mathbf{2}^{-}}$ Lösung sofort in grün um. Der Farbenwechsel ist durch eine Umsetzung von $\mathrm{CuBr}_{2}$ in $\mathrm{Cu}(\mathrm{OCN})_{2}$, welch letzteres in alkoholischer Lösung grün ist, bedingt. Gleichzeitig wird $\mathrm{HgBr}_{2}$ gebildet. Wenn bei der Reaktion Aceton oder Methyläthylketon als Lösungsmittel gebraucht werden, so fällt das gebildete Cu$(\mathrm{OCN})_{2}$ zum grössten Teil aus und kann nach Waschen mit den Ketonen und schliesslich mit Äther als lichtgrünes mikrokristallines Pulver isoliert werden. Das reinste Produkt wurde aus Aceton erhalten. In dieser Weise ist das aus wässriger oder alkoholischer Lösung wegen solvolytischer Zersetzung nicht gewinnbare Cyanat leicht darstellbar. Wie von Naumann ${ }^{9}$ beobachtet worden ist, setzt sich $\mathrm{HgCl}_{2}$ mit $\mathrm{CuBr}_{2}$ in Aceton um, wobei zunächst $\mathrm{CuCl}_{2}$ gebildet, aber allmählich zu $\mathrm{CuCl}$ reduziert wird. Wie ich selbst konstatiert habe, tritt in Methanol und Äthanol derselbe momentane Farbenumschlag ein wie mit $\mathrm{Hg}(\mathrm{OCN})_{2}$.

Das Mercurichlorid wirkt bekanntlich auf viele organische Verbindungen merkurierend, wobei Quecksilber an Kohlenstoff fixiert wird. Die Ähnlichkeit zwischen Chlorid und Cyanat lässt vermuten, dass wenigstens in gewissen Fällen das Chlorid bei solchen Reaktionen durch das Cyanat vertreten werden kann. So wird, wie ich festgestellt habe, Pyrrol in Wasser glatt durch $\mathrm{Hg}$ $(\mathrm{OCN})_{2}$ zu einer Verbindung $\mathrm{C}_{4} \mathrm{H}_{2} \mathrm{NH}(\mathrm{HgOCN})_{2}$ merkuriert unter gleichzeitiger Entwicklung von Cyansäure. Thiophen wird von einer acetatgepufferten Wasserlösung von $\mathrm{Hg}(\mathrm{OCN})_{2}$ eben wie von $\mathrm{HgCl}_{2}$ langsam angegriffen. Isoliert wurden durch Extraktion mit Aceton ein Monomerkurierungsprodukt und ein dimerkuriertes, deren Untersuchung jedoch noch nicht abgeschlossen ist. Ersteres, welches durch seine Ätherlöslichkeit leicht von letzterem separiert werden kann, kristallisiert aus Äther in Prismen von Schmelzp. $105^{\circ}$.

\section{Mercurocyanat}

Mercurocyanat scheint erstmalig von Wöhler ${ }^{10}$ dargestellt zu sein, er gewann es durch Umsetzen von $\mathrm{Hg}_{2}(\mathrm{NO})_{2}$ mit $\mathrm{Ba}(\mathrm{OCN})_{2}$. Das Salz ist sehr wenig untersucht worden. Von Knoll \& Co. ${ }^{11}$ ist es für Darstellung von $\alpha$-Bromiso- 
valerylharnstoff gebraucht worden und von Rao und Watson ${ }^{8}$ für die Synthese von $\mathrm{Br} \cdot \mathrm{Hg}(\mathrm{OCN})$ und $\mathrm{J} \cdot \mathrm{Hg}(\mathrm{OCN})$. Wie ich festgestellt habe, kann das Salz durch Fällen einer halbmolaren mit $\mathrm{HNO}_{3}$ angesäuerten Wasserlösung von $\mathrm{Hg}_{2}\left(\mathrm{NO}_{3}\right)_{2}$ mit $\mathrm{NaOCN}$ in einigem Úberschuss in $95 \%$-iger Ausbeute und von grosser Reinheit erhalten werden. Es ist in heissem Wasser etwas löslich und daraus bei Abkühlen als kleine Nadeln erhältlich. Seine Dissoziation in Wasser in der Wärme sowie die umgekehrte Reaktion bei Zimmertemperatur sind schon erwähnt worden, ebenso seine Bildung nach der Ederschen Reaktion. Wässriges $\mathrm{NH}_{3}$ bewirkt sofortige Schwärzung durch Bildung von met. $\mathrm{Hg}$.

\section{Kupfercyanate}

Normale Cyanate von Kupfer waren meines Wissens bis jetzt unbekannt *, woran die leichte Hydrolysierbarkeit hauptsächlich die Schuld trug. Wie Cupricyanat aus Acetonlösung darzustellen ist, wurde oben mitgeteilt. Ein Doppelsalz mit Kaliumcyanat kann aber in Wasser dargestellt werden; es fällt bei Vermischen einer konzentrierten $\mathrm{Cu}\left(\mathrm{NO}_{3}\right)_{2}$-Lösung mit KOCN in Ứberschuss als ein voluminöser Kristallniederschlag aus. Das Salz, welches die Formel $\mathrm{KCu}(\mathrm{OCN})_{3}$ hat, löst sich in Wasser mit blauer Farbe, die Löslichkeit beträgt bei $20^{\circ} 5,2 \mathrm{~g}$ pro $100 \mathrm{ml}$ (durch Cu-Analyse einer abgewogenen Menge gesättigter Lösung bestimmt). In absolutem Alkohol ist die Löslichkeit so gering, dass der Alkohol farblos bleibt. Aceton löst kleine Mengen mit hellgrüner Farbe. Die Verbindung kann nur kurze Zeit in Wasserlösung aufbewahrt werden, da sie rasch unter Bildung von basischem Cyanat zersetzt wird. Versuche ein entsprechendes Natriumsalz zu bereiten scheiterten, wahrscheinlich wegen einer zu grossen Löslichkeit des Salzes in Wasser.

Die Fähigkeit Doppelcyanate zu bilden ist auch für das Cuprocyanat CuOCN eigentümlich. Wenn eine Wasserlösung von Kupfersulfat oder Nitrat mit überschüssigem Kaliumcyanat und dann mit Kaliumbisulfit versetzt wird, tritt bald Ausfällung von glänzenden farblosen Kristallblättern ein, und gleichzeitig beginnt eine ziemlich lebhafte Entwicklung von $\mathrm{CO}_{2}$. Die Zusammensetzung der Kristalle ist $\mathrm{KCu}_{3}(\mathrm{OCN})_{4}$. Bei später ausgeführten Synthesen wurde immer als $\mathrm{Cu}^{2+}{ }_{-} \mathrm{Salz}$ das Nitrat verwendet und das Bisulfit gegen eine frisch bereitete Lösung von $\mathrm{SO}_{2}$ in Wasser ausgetauscht. Die Lösung muss dann um die Auskristallisation zu beschleunigen gepuffert werden. Die Pufferung ist auch dadurch geboten, dass die Zersetzung der bei der Reduktion gebildeten Cyansäure in $\mathrm{CO}_{2}$ und $\mathrm{NH}_{4}$-Salz unbedingt verhindert werden muss um Komplikationen durch Doppelsalzbildung von $\mathrm{NH}_{4} \mathrm{OCN}$ mit $\mathrm{CuOCN}$ zu vermeiden. Als Puffer wurde Kaliumacetat benutzt, wodurch die $\mathrm{CO}_{2}$-Bildung praktisch vollständig zurückgedrängt werden konnte. Nach neuesten Messungen von Amell ${ }^{\mathbf{1 2}}$ ist die thermodynamische Dissoziationskonstante der Cyansäure bei $20^{\circ} 2,0 \times 10^{-4}$, diejenige von Essigsäure ist bekanntlich ungefähr ein Zehntel von diesem Wert.

* In Williams, H. E. Cyanogen Compounds, 2. Aufl. s. 73, wird Cupricyanat als eine grüne in Wasser unlösliche Verbindung beschrieben. Wie ich festgestellt habe, handelt es sich hier um ein basisches Cyanat von der Formel $\mathrm{Cu}(\mathrm{OH})_{2} \cdot \mathrm{Cu}(\mathrm{OCN})_{2}$. 
Dieselbe Zusammensetzung wie das Kaliumsalz hat das in analoger Weise dargestellte Rubidiumsalz, also $\mathrm{RbCu}_{3}(\mathrm{OCN})_{4}$, während diejenige der Natriumverbindung der Formel $\mathrm{NaCu}_{4}(\mathrm{OCN})_{5}$ gehorcht. Auch dieses Salz kristallisiert in Tafeln *. Selbstverständlich ist bei Synthesen der Na- und Rb-Verbindungen Na- bezw. Rb-Acetat als Puffer zu verwenden.

Die Komplexizität dieser in Wasser schwerlöslichen Doppelcyanate ist schwach; sie werden z.B. von Wasser unter Bildung von gelbem $\mathrm{Cu}_{2} \mathrm{O}$ hydrolytisch zersetzt.

Die abweichende Formel des Na-Salzes gab mir den Anlass, auch die Darstellung eines Lithiumsalzes zu versuchen. Bei Zusatz von Lithiumcyanat zu einer Mischung von $\mathrm{Cu}\left(\mathrm{NO}_{3}\right)_{2}, \mathrm{LiOAc}$ und $\mathrm{SO}_{2}$ trat in einigen Fällen keine, in anderen eine geringfügige Kristallisation ein, bei Einleiten von $\mathrm{SO}_{2}$ jedoch eine reichliche. Die Analyse der gewaschenen und getrockneten prismatischen Kristalle zeigte, dass sie alkalifrei waren und die Formel CuOCN hatten. LiOCN ist also zur Bildung von schwerlöslichem Doppelcyanat unfähig, und damit war also eine Methode zur Darstellung von CuOCN gefunden.

Das Cuprocyanat ist in seinem chemischen Verhalten Cuprochlorid sehr ähnlich. Es wird wie dieses leicht von Wasser under Gelbfärbung hydrolysiert und in feuchtem Zustand leicht durch den Luftsauerstoff unter Grünfärbung oxydiert. Dieses ist auch bei seinen Doppelverbindungen der Fall, weshalb das Abfiltrieren und Auswaschen in einer Stickstoffatmosphäre auszuführen ist. Brom in Wasser bewirkt sofortige Auflösung zu $\mathrm{CuBr}_{2}$, und $\mathrm{Cu}(\mathrm{OCN})_{2},(\mathrm{SCN})_{2}$ in einem organischen Solvenz schwärzt unter Bildung von $\mathrm{Cu}(\mathrm{SCN})_{2}$. Konzentrierte Wasserlösungen von KOCN lösen CuOCN zu einer leicht oxydablen Lösung. Längeres Aufbewahren geschieht am besten in Vakuum unter Ausschluss von Tageslicht. CuOCN wirkt ferner als Katalysator nach Sandmeyer bei Einführung von einer Isocyanatgruppe in den Benzolkern, wovon weiter unten die Rede sein wird.

Die für die Synthesen von $\mathrm{RbCu}_{3}(\mathrm{OCN})_{4}$ und $\mathrm{CuOCN}$ erforderlichen $\mathrm{Rb}$ bezw. Li-Cyanate sind aus den entsprechenden Sulfaten durch Umsatz mit Bariumcyanat und Konzentrieren der filtrierten Lösung durch Vakuumdestillation erhältlich. Die Methoden der Literatur zur Herstellung von $\mathrm{Ba}(\mathrm{OCN})_{2}$ sind wenig befriedigend; das Salz kann aber, wie ich gefunden habe, sehr bequem durch Fällen einer gesättigten wässrigen Lösung von Natriumcyanat mit einer äquivalenten Menge von Bariumrhodanid- oder Perchlorat in Methanol gewonnen werden **. Das Cyanat kristallisiert anhydrisch in kleinen Prismen von neutraler Reaktion, seine Löslichkeit in Wasser bei $20^{\circ}$ wurde durch Ba-Analyse der gesättigten Lösung zu $10,6 \mathrm{~g}$ pro $100 \mathrm{ml}=0,45 \mathrm{M}$ bestimmt.

Wie schon oben erwähnt, zeigt sich die Halogenähnlichkeit der Cyanatgruppe auch darin, dass CuOCN als Sandmeyerkatalysator bei der Einführung von einer Isocyanatgruppe in den Benzolkern wirken kann. Für eine solche Reaktion via ein Diazoniumsalz war man früher auf Katalyse nach Gattermann ${ }^{14}$ also mit metallischem $\mathrm{Cu}$ hingewiesen.

* Von Wöhler und Berthmann ${ }^{13}$ sind Natriumcuprofulminate der Formeln $\mathrm{NaCu}(\mathrm{ONC})_{\text {r }}$ und $\mathrm{Na}_{2} \mathrm{Cu}(\mathrm{ONC})_{3} 3 \mathrm{H}_{2} \mathrm{O}$ dargesteilt worden.

** Versuche analog das Strontiumcyanat aus dem Rhodanid und NaOCN zu gewinnen scheiterten, wahrscheinlich wegen einer zu grossen Löslichkeit des Salzes in Methanol-Wasserlösung. 
Wird zu einer diazotierten Lösung von Anilinhydrosulfat KOCN gesetzt und dann sofort $\mathrm{KCu}_{3}(\mathrm{OCN})_{4}$ oder $\mathrm{NaCu}_{4}(\mathrm{OCN})_{5}$, tritt unmittelbar eine lebhafte Stickstoffentwicklung ein, und es scheidet sich $\mathrm{C}_{6} \mathrm{H}_{5} \mathrm{NCO}$ als Öltropfen ab, die mit einem passenden Solvenz wie Benzol, Chloroform oder Tetrachlorkohlenstoff aufgenommen werden können. Die Reaktion ist wegen der Wasserempfindlichkeit des Isocyanats etappenweise auszuführen; der Katalysator ist in Portionen zuzusetzten und das Reaktionsprodukt jedesmal zu extrahieren. Die Ausbeuten sind aus dem genannten Grunde nicht besonders gut, auch nicht bei möglichst rapidem Arbeiten. Etwas bessere Ausbeuten, bis auf $30 \%$, sind mit $o$-Toluidin erhalten worden, was wohl hauptsächlich der geringeren Wasserempfindlichkeit des o-Tolylisocyanats zuzuschreiben ist.

Das durch Fraktionieren isolierte Reaktionsprodukt wurde durch kurzes Erwärmen mit $o$-Kresol identifiziert, wobei in fast quantitativer Ausbeute der $o$-Tolylester der o-Tolyl-Carbaminsäure entstand.

\section{EXPERIMENTELLER TEIL}

Das für die folgenden Versuche benutzte Kaliumcyanat war Bakers' Analyzed ebenso das Cuprinitrat; das Natriumcyanat war von Hopkin und Williams geliefert.

\section{Cadmiumcyanat aus Sulfat und Bariumcyanat}

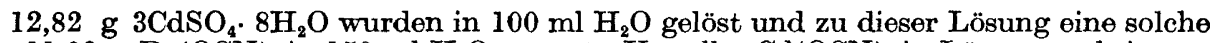
von $11,09 \mathrm{~g} \mathrm{Ba}(\mathrm{OCN})_{2}$ in $150 \mathrm{ml} \mathrm{H}_{2} \mathrm{O}$ gesetzt. Um alles $\mathrm{Cd}(\mathrm{OCN})_{2}$ in Lösung zu bringen, wurde auf ein Volumen von $550 \mathrm{ml}$ verdünnt. Das Reaktionsgemisch blieb über Nacht bei Zimmertemperatur stehen, wonach der Niederschlag von $\mathrm{BaSO}_{4}$ auf einem grossen Jenaer Glasfilter G4 abfiltriert wurde. Durch Vakuumdestillation wurde dann die Lösung sofort auf ein Volumen von $55 \mathrm{ml}$ konzentriert, wobei Kristallisatiou in seideglänzenden Blättern eintrat. Nach etwa zweistündigem Kühlen in Eisbad wurde die Mutterlauge abgesogen, die Kristalle zunächst mit Aceton und dann mit Äther gewaschen und kurz an der Luft getrocknet. Gewicht $10,0 \mathrm{~g}$ statt theor. 12,0. Das so präparierte Salz war mit etwas basischem Salz gemengt und löste sich nicht klar in Wasser. Zwecks Reinigung wurden $2,2 \mathrm{~g}$ in $100 \mathrm{ml} \mathrm{H} \mathrm{H}_{2} \mathrm{O}$ gelöst und soviel einer $0,25 \mathrm{M}$ Essigsäurelösung zugesetzt, dass die Trübung verschwand. Es wurde nun sofort durch Vakuumdestillation mit einer Badtemperatur, die nicht $50^{\circ}$ überstieg, auf ein Volumen von $25 \mathrm{ml}$ eingeengt und dann nach Zugabe von $3 \mathrm{ml} \mathrm{0,25} \mathrm{M} \mathrm{HOAc} \mathrm{in} \mathrm{Eisbad} \mathrm{der} \mathrm{Kristallisation} \mathrm{überlassen.} \mathrm{Nach} \mathrm{etwa} 3$ Stunden Abfiltrieren, Waschen und Trocknen, wie oben angegeben. Ausbeute $1,6 \mathrm{~g}=$ $73 \%$.

Das Cd wurde als Oxid bestimmt, $\mathrm{C}$ und $\mathrm{H}$ nach Liebig (Gef. Cd 46,64; C 9,96; H 2,08. Ber. für 2Cd(OCN $)_{2} 5 \mathrm{H}_{2} \mathrm{O}$ [482,92): $\mathrm{Cd} 46,55 ; \mathrm{C} \mathrm{9,94;} \mathrm{H} \mathrm{2,08;} \mathrm{für} \mathrm{Cd}(\mathrm{OCN})_{2} 2 \mathrm{H}_{2} \mathrm{O}(232,45)$ : $\mathrm{Cd} 48,35 ; \mathrm{C} 10,32 ; \mathrm{H} 1,73)$. Bei Aufbewahren in Vakuum über konz. $\mathrm{H}_{2} \mathrm{SO}_{4}$ gibt das Salz langsam sein Kristallwasser ab. 1

\section{Cadmiumcyanat aus Jodid undISilbercyanat}

$21,976 \mathrm{~g} \mathrm{CdJ}_{2}$ wurden in $100 \mathrm{ml}$ reinem Aceton suspendiert und zu der Lösung 17,988 $\mathrm{g}$ AgOCN in Portionen gesetzt. Beide Präparate waren von hochgradiger Reinheit. Reaktion unter Wärmeentwicklung. Das Gemisch blieb während 2 Tagen bei Zimmertemperatur unter zeitweiligem Umschütteln stehen. Dann wurde der Niederschlag abfiltriert, in Vakuum getrocknet, pulverisiert und dann wieder in die Acetonlösung hineingebracht. Nach weiteren 2 Tagen war die Lösung jodidfrei, der Niederschlag wurde abfiltriert, mit Äther gewaschen und in Vakuum getrocknet. Gewicht 41,7 statt 39,9 g. Zwecks Gewinnung des Cyanats wurden 6,66 $\mathrm{g}$ des Gemenges von $\mathrm{AgJ}$ und anhydrischem $\mathrm{Cd}(\mathrm{OCN})_{2}$ mit $115 \mathrm{ml}$ Wasser digeriert, die Wasserlösung abgesogen, und der Niederschlag mit

Acta Chem. Scand. 11 (1957) No. 10 
$2 \times 10 \mathrm{ml}$ Wasser gewaschen. Gewicht nach Trocknen 4,73 statt 4,70 g. Durch Konzentrierung der Lösung wie oben angegeben auf ein Volumen von $25 \mathrm{ml}$ und nachfolgender Kühlung konnten 1,43 g Ag-freies Cyanat isoliert werden.

\section{Darstellung von Mercuricyanat aus $\mathrm{HgCl}_{2}$ und $\mathrm{AgOCN}$} in $\ddot{A} t h e r$.

$27,25 \mathrm{~g}$ gepulvertes $\mathrm{HgCl}$, wurden in $600 \mathrm{ml}$ trocknem Äther gelöst und $33 \mathrm{~g}$ reines AgOCN zugesetzt (theor. 30,0). Das Reaktionsgemisch wurde während einer Stunde geschüttelt und dann während eines Tages sich selbst überlassen. Die Lösung war dann chlorfrei. Nach Filtrieren wurde der Äther abdestilliert, wobei Kristallisation von $\mathrm{Hg}(\mathrm{OCN})_{2}$ eintrat. Der von $\mathrm{AgCl}, \mathrm{AgOCN}$ und $\mathrm{Hg}(\mathrm{OCN})$, bestehende Niederschlag wurde dann erschöpfend mit Äther extrahiert, wodurch insgesamt $27 \mathrm{~g}$ reines $\mathrm{Hg}(\mathrm{OCN})_{2}$ erhalten wurden. Das Präparat ist in einer trocknen Atmosphäre, am besten in Vakuum aufzubewahren. Bei der Analyse wurde $\mathrm{Hg}$ als $\mathrm{HgS}$ bestimmt, $\mathrm{N}$ nach Kjeldahl und $\mathrm{C}$ auf nassem Wege durch Verbrennung mit einem Gemisch von $\mathrm{H}_{2} \mathrm{SO}_{4}, \mathrm{CrO}_{3}, \mathrm{H}_{3} \mathrm{PO}_{4}$ und $\mathrm{KJO}_{2}$. (Gef. Hg 70,66; N 9,80; C 8,36. Ber. für $\mathrm{Hg}(\mathrm{OCN})_{2}(284,61): \mathrm{Hg} \mathrm{70,49;} \mathrm{N} \mathrm{9,84;} \mathrm{C} \mathrm{8,44).}$ Die Dichte wurde pyknometrisch bei $20^{\circ} \mathrm{zu} \mathrm{4,35} \pm 0,01$ mit Brombenzol als Sperrflüssigkeit bestimmt.

\section{Darstellung von Mercuricyanat aus Quecksilber- oxyd und Cyansäure}

In $75 \mathrm{ml}$ eisgekühltem trocknem Äther wurden $8,9 \mathrm{~g}$ trocknes $\mathrm{HCl}$ eingeleitet und zu der Lösung in Portionen $47 \mathrm{~g} \mathrm{AgOCN}$ gegeben (theor. 36,9). Reaktion unter Wärmeentwicklung. Nach Umschütteln unter Kühlung während etwa 1,5 Stunden wurde der Niederschlag auf einem Glasfilter G3 mit $65 \mathrm{ml}$ Xther in Portionen ausgewaschen und zu der Lösung sofort 24,4 g gelbes, in der Kälte ausgefälltes, mit Wasser, Alkohol und Äther gewaschenes $\mathrm{HgO}$ in Portionen unter Umschütteln gesetzt. Reaktion unter einiger Wärmeentwicklung, weshalb anfänglich etwas mit kaltem Wasser gekühlt wurde. Beim Stehen wurde die gelbe Farbe des Bodenkörpers allmählich blasser und war nach 12 Stunden gelblich weiss. Der stechende Geruch von HOCN war nun verschwunden. Der Bodenkörper wurde abfiltriert, mit etwas Äther gewaschen und in Vakuum getrocknet. Gewicht 23,4 g. Die Mutterlauge wurde durch Abdestillieren des Äthers konzentriert, wobei Kristallisation eintrat. Die Kristalle wurden sofort von dem wasserhaltigen Äther abfiltriert und in Vakuum getrocknet. Die 23,4 $\mathrm{g}$ Bodensubstanz wurden mit Äther erschöpfend extrahiert und insgesamt konnten nach Konzentrieren der Lösungen 21,7 g

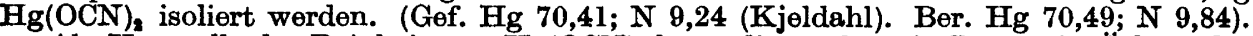

Als Kontrolle der Reinheit von $\mathrm{Hg}(\mathrm{OCN})_{2}$ kann die restlose Auflösung in Äther oder Aceton benutzt werden. Wenn sich eine Probe nicht vollständig löst, ist eine Umlösung in Äther für die Reinigung genügend.

\section{Darstellung von Mercurocyanat}

$56,1 \mathrm{~g}(0,1 \mathrm{Mol}) \mathrm{Hg}_{2}\left(\mathrm{NO}_{3}\right)_{2} \cdot 2 \mathrm{H}_{2} \mathrm{O}$ wurden in einer Mischung von $150 \mathrm{ml} \mathrm{H}_{2} \mathrm{O}$ und $20 \mathrm{ml} 2 \mathrm{M} \mathrm{HNO}_{3}$ bei gelinder Wärme aufgelöst, die Lösung mit Eis gekühlt und sofort mit einer Lösung von 17,5 $\mathrm{g} \mathrm{NaOCN}$ in $200 \mathrm{ml} \mathrm{H}_{8} \mathrm{O}$ gemischt, Die Menge von $\mathrm{NaOCN}$ war so bemessen, dass sie ausser zur Ausfüllung von $\mathrm{Hg}_{3}(\mathrm{OCN})_{2}$ auch zur Pufferung der überschüssigen $\mathrm{HNO}_{3}$ ausreichte. Es entstand sofort ein rein weisser Niederschlag, der sich ziemlich rasch absetzte. Nach einer Viertelstunde wurde der Niederschlag auf einem grösseren Jenaer Glasfilter G3 abfiltriert, durch Umrühren mit eiskaltem Wasser viermal ausgewaschen, dann zweimal mit Alkohol und Äther und zuletzt in Vakuum über $\mathrm{H}_{2} \mathrm{SO}_{4}$ getrocknet. Gewicht 46,2 statt 48,5 $\mathrm{g}=95 \%$. (Gef. $\mathrm{Hg} 82,90$. Ber. für $\mathrm{Hg}_{2}(\mathrm{OCN})_{2}$ (485,22): $\mathrm{Hg}$ 82,68). 


\section{Dissoziation von Mercurocyanat durch Erhitzen $\mathrm{mit}$ W as ser}

$41,9 \mathrm{~g} \mathrm{Hg}_{2}(\mathrm{OCN})_{2}$ wurden mit $200 \mathrm{ml}$ siedeheissem Wasser kurz umgeschüttelt, wobei der Niederschlag sich sofort grau färbte. Die warme Lösung wurde durch ein grosses Glasfilter G3 filtriert und sofort gekühlt, wobei eine kleine Menge einer in kleinen Nadeln anschiessenden Substanz sich absetzte, $\left(\mathrm{Hg}_{2}(\mathrm{OCN})_{2}\right)$. Das Digerieren wurde nun mit neuen Portionen von $200 \mathrm{ml}$ heissem Wasser fortgesetzt, bis bei Ankühlen keine weitere Kristallisation von $\mathrm{Hg}_{2}(\mathrm{OCN})_{2}$ eintrat. Der rückständige Niederschlag konnte nach Trocknen praktisch vollständig $\mathrm{zu}$ flüssigem $\mathrm{Hg}$ zusammengerieben werden. Gewicht 15,8 statt $16,9 \mathrm{~g}$. Die vereinigten völlig klaren Wasserextrakte, deren Volumen etwa 1 Liter betrug, wurden möglichst rasch durch Vakuumdestillation konzentriert, wobei Kristallisation allmählich eintrat. Die Kristalle wurden fraktioniert aufgesammelt und in Vakuum getrocknet, und die Konzentrierung der Mutterlauge bis zu wenigen $\mathrm{ml}$ getrieben. Insgesamt konnten $20,2 \mathrm{~g}$ Substanz statt theor. $24 \mathrm{~g}$ isoliert werden und daraus nach Umlösen in Äther $18,4 \mathrm{~g}$ reines $\mathrm{Hg}(\mathrm{OCN})_{2}$.

\section{Reduktion von $\mathrm{Hg}(\mathrm{OCN})_{2}$ z u $\mathrm{Hg}_{2}(\mathrm{OCN})_{2}$}

Lösungsmittel Wasser. $2,84 \mathrm{~g} \mathrm{Hg}(\mathrm{OCN})_{2}$ wurden in $50 \mathrm{ml} \mathrm{H}_{2} \mathrm{O}$ gelöst und während einer Stunde mit $33 \mathrm{~g}$ met. $\mathrm{Hg}$ geschüttelt. Probe auf $\mathrm{Hg}^{2}+$ mit $\mathrm{NaOH}$ gab dann keinen Niederschlag von HgO. Nach Absaugen der Wasserlösung wurde mit Wasser, Alkohol und Äther gewaschen und luftgetrocknet. $29 \mathrm{~g} \mathrm{Hg}$ wurden entfernt und der Rückstand fünfmal mit je $25 \mathrm{ml}$ siedeheissem Wasser ausgelaugt, der wässrige Extrakt eisgekühlt, wobei $0,11 \mathrm{~g} \mathrm{Hg}_{2}(\mathrm{OCN})_{2}$ ausfiel. Die Mutterlauge wurde durch Vakuumdestillation eingetrocknet und dabei ein Salzrückstand von 2,33 g erhalten. Dieser löste sich bei Digerieren mit $170 \mathrm{ml}$ Äther mit einem äusserst kleinen gelben Rückstand. $1,82 \mathrm{~g} \mathrm{Hg}(\mathrm{OCN})_{2}$ wurden aus der Ätherlösung isoliert.

Lösungsmittel Äther. 4,40 $\mathrm{g} \mathrm{Hg}(\mathrm{OCN})_{2}$ wurden in $100 \mathrm{ml}$ Ather suspendiert und mit $125 \mathrm{~g}$ met. Hg geschüttelt. Die Reduktion war nach 2 Stunden beendet, da die Probe mit $\mathrm{NaOH}$ einen negativen Ausfall hatte. Der Niederschlag wurde abfiltriert, wie oben beschrieben gewaschen und getrocknet und mit $3 \times 50 \mathrm{ml}$ heissem Wasser ausgelaugt. Beim Einengen der filtrierten Lösung konnten 3,0 g Mercuricyanat isoliert werden. Die Ätherlösung, wo die Reduktion ausgeführt wurde, gab keinen Eindampfungsrückstand, was also zeigt dass der Reaktionsverlauf ein quantitativer war.

\section{Cupricyanat}

$6,2 \mathrm{~g} \mathrm{Hg}(\mathrm{OCN})_{2}$ wurden in $50 \mathrm{ml}$ Aceton gelöst und zu der Lösung 4,46 $\mathrm{g}$ anhydrisches $\mathrm{CuBr}_{2}$ gesetzt. Unter gelinder Wärmeentwicklung geht das Bromid in Lösung und reagiert mit dem Cyanat unter Ausfällung eines hellgrünen Niederschlags. Die Umsetzung ist nach wenigen Minuten beendet*. Nach stundenlangem Stehen bei Zimmertemperatur wird der Niederschlag unter Ausschluss von Feuchtigkeit abfiltriert, dreimal mit kleinen Mengen des Ketons unter Umrührung und dreimal mit trocknem Äther gewaschen und dann in Vakuum über $\mathrm{P}_{2} \mathrm{O}_{5}$ bis zu Gewichtskonstanz getrocknet. Ausbeute 2,6 $\mathrm{g}$ statt theor. 2,95. (Gef. Cu 42,34; $\mathrm{C} 16,62$. Ber. für $\left.\mathrm{Cu}(\mathrm{OCN})_{2}(147,59): \mathrm{Cu} 43,08 ; \mathrm{C} 16,27\right)$.

$\mathrm{Cu}(\mathrm{OCN})_{2}$ ist leicht und vollständig löslich in Wasser zu einer blauen Lösung, die aber bald unlösliches basisches Salz absetzt. In Methanol und Äthanol sowie in Aceton ist es mit grüner Farbe etwas löslich. Diese Lösungen sind bei längerem Aufbewahren auch nicht stabil. Die Dichte wurde bei $20^{\circ}$ pyknometrisch mit Xylol als Sperrflüssigkeit zu 2,45士 0,01 bestimmt.

* In rigorös getrocknetem Aceton ist die Reaktion träger

Acta Chem. Scand. 11 (1957) No. 10 


\section{Kaliumcupricyanat}

Eine Lösung von 8,2 $\mathrm{g}$ KOCN in $25 \mathrm{ml} \mathrm{H}_{2} \mathrm{O}$ wurde durch Zusatz von 0,2 $\mathrm{g} \mathrm{Ba}\left(\mathrm{NO}_{3}\right)_{2}$ und Abfiltrieren des Niederschlags von Carbonat befreit, und die eisgekühlte Lösung mit einer ebenfalls eisgekühlten Lösung von $6,0 \mathrm{~g} \mathrm{Cu}\left(\mathrm{NO}_{3}\right)_{2} .3 \mathrm{H}_{2} \mathrm{O}$ in $15 \mathrm{ml} \mathrm{H}_{2} \mathrm{O}$ gemischt. Es entstand sofort ein aus feinen Nadeln bestehen:der hellgrüner Kristellbrei. Nach etwa 30 Min wurden die Kristalle auf einem Glasfilter G3 aufgesammelt, mit 10 ml eiskaltem Wasser und dann mit Alkohol und Äther unter Umrühren gewaschen und in Vakuum über konz. $\mathrm{H}_{2} \mathrm{SO}$, getrocknet. Ausbeute 4,2 g. Bei der Analyse wurde $\mathrm{K}$ als Sulfat und $\mathrm{Cu}$ als Rhodanur bestimmt. (Gef. K 16,51; $\mathrm{Cu} 27,74$. Ber. für $\mathrm{KCu}(\mathrm{OCN})_{3}(228,62)$ : $\mathrm{K}$ 17,09: $\mathrm{Cu}$ 27,86).

\section{Kalium cuprocyanat}

$12,0 \mathrm{~g} \mathrm{Cu}\left(\mathrm{NO}_{3}\right)_{2} \cdot 3 \mathrm{H}_{2} \mathrm{O}$ wurden in $50 \mathrm{ml}$ Wasser gelöst und dazu $50 \mathrm{ml}$ einer 1,3 $\mathrm{M}$

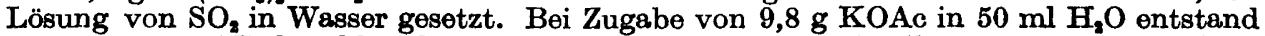
ein gelbgrüner Niederschlag der sich sofort auflöste, wenn eine Lösung von 8,1 g KOCN in $50 \mathrm{ml} \mathrm{H} \mathrm{H}_{2} \mathrm{O}$ zugegeben wurde. Die grüne Farbe der Lösung wurde rasch abgeblasst, und Ausfällung von glänzenden Kristallblättern setzte sofort ein. Das Gemisch wurde 45 Min im Eisbad gekühlt und denn die Kristalle auf ein Glasfilter von mittlerer Feinheit aufgesammelt, mit $0,2 \mathrm{M} \mathrm{SO}$-Lösung dreimal unter Umrührung gewaschen, denn zweimal mit Alkohol und dreimal mit Âther und dann sofort in Vakuum über $\mathrm{H}_{2} \mathrm{SO}$, getrocknet. Ausbeute 5,4 statt theor. 6,6 g. Abfiltrierung und Auswaschen sind in einer $\mathrm{N}_{2}$-Atmosphäre auszuführen, und bevor der Zugabe von Alkohol ist das $\mathrm{SO}_{2}$ mit $\mathrm{N}_{2} \mathrm{zu}$ verjagen, da alkoholisches $\mathrm{SO}_{2}$ mit CuOCN eine braune Färbung gibt.

Bei der Analyse wurde $\mathrm{K}$ als Sulfat nach Entfernen des $\mathrm{Cu}$ mit $\mathrm{H}_{2} \mathrm{~S}$ als $\mathrm{Cu}_{2} \mathrm{~S}$ bestimmt. Für die Cu-Analyse wurde die Probe in Wasser suspendiert; soviel Brom wurde zugesetzt, dass vollständige Auflösung eintrat, und dann nach $\mathrm{Zugabe}$ von $\mathrm{SO}_{2}$ wurde $\mathrm{Cu}$ als $\mathrm{CuSCN}$ ausgefällt und gewogen. (Gef. K 9,42; Cu 47,37; C 12,29; H 0,19. Ber. für $\mathrm{KCu}_{3}(\mathrm{OCN})_{4}$ $(397,85):$ K 9,83; Cu 47,94; C 12,07).

\section{Natriumcuprocyanat}

$12,1 \mathrm{~g} / \mathrm{Cu}\left(\mathrm{NO}_{3}\right)_{2} .3 \mathrm{H}_{2} \mathrm{O}$ in $25 \mathrm{ml} \mathrm{H} \mathrm{H}_{2} \mathrm{O}+50 \mathrm{ml}$ einer $1,4 \mathrm{M} \mathrm{SO}_{2}$-Lösung $+17 \mathrm{~g} \mathrm{NaOAc}$. $3 \mathrm{H}_{2} \mathrm{O}$ in $50 \mathrm{ml}+6,5 \mathrm{~g} \mathrm{NaOCN}$ in $75 \mathrm{ml}$. Auskristallisation etwas langsamer als beim Kaliumsalz. Kristallisationszeit 1 Stunde. Auswaschen und Trocknen wie oben beschrieben mit der Ausnahme, dass statt mit Alkohol mit einer 0,1 M Lösung von HOAc in Alkohol gewaschen wurde. Ausbeute 4,5 g statt 6,1. Ziemlich grosse rein weisse Blätter. Das Na wurde als Sulfat, Cu als CuSCN bestimmt. (Gef. $\mathrm{Na} \mathrm{4,68;} \mathrm{Cu} \mathrm{51,80.} \mathrm{Ber} \mathrm{für}$ $\mathrm{NaCu}_{4}(\mathrm{OCN})_{5}(487,32): \mathrm{Na} 4,72 ; \mathrm{Cu} \mathrm{52,12)}$.

\section{Rubidiumcuprocyanat}

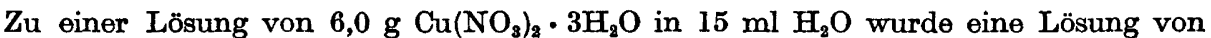
RbOAc, aus 6,52 $\mathrm{g} \mathrm{Rb}_{2} \mathrm{CO}_{3}$ und $3,25 \mathrm{ml}$ Eisessig in $25 \mathrm{ml} \mathrm{H}_{2} \mathrm{O}$ dargestellt, gesetzt und dann eine Lösung von $\mathrm{RbOCN}$, die aus 6,52 $\mathrm{g} \mathrm{Rb}_{2} \mathrm{SO}_{4}$ und 5,4 $\mathrm{g} \mathrm{Ba}(\mathrm{OCN})_{2}$ in $100 \mathrm{ml} \mathrm{H}_{2} \mathrm{O}$ bereitet und dann durch Vakuumdestillation auf ein Volumen von 25 ml konzentriert worden war. Kleine Mengen eines hellgrünen Niederschlages entstanden, die aber bei Zusatz von $25 \mathrm{ml}$ einer $1,5 \mathrm{M} \mathrm{SO}$-Lösung sofort verschwanden. Rasches Ausfallen eines weissen Niederschlags von kleinen Schuppen. Nach halbstündigem Stehen in Eisbad Abfiltrieren und Waschen wie bei der Na-Verbindung und Trocknen in Vakuum. Gewicht $3,3 \mathrm{~g}$, theor. 3,9 g. Die Mutterlauge setzte bei Stehen noch etwa $0,1 \mathrm{~g}$ Substanz als grössere Tafeln mit rhombischer Begrenzung ab. Bei der Analyse wurde Rb als Perchlorat und $\mathrm{Cu}$ als CuSCN bestimmt. (Gef. $\mathrm{Rb}$ 18,89; $\mathrm{Cu} 43,19$. Ber. für $\mathrm{RbCu}_{3}(\mathrm{OCN})$ ( 444,23$)$ : $\mathrm{Rb}$ 19,24; $\mathrm{Cu} 42,93)$.

$$
\text { Cuprocyanat }
$$

$24,2 \mathrm{~g} \mathrm{Cu}\left(\mathrm{NO}_{3}\right)_{2} \cdot 3 \mathrm{H}_{2} \mathrm{O}$ in $25 \mathrm{ml} \mathrm{H} \mathrm{H}_{2} \mathrm{O}+0,3 \mathrm{Mol} \mathrm{LiOAc}$ in $60 \mathrm{ml} \mathrm{H}_{2} \mathrm{O}$, wobei eine dunkelblaue Lösung entstand. $\mathrm{Zu}$ dieser wurde eine Lösung von $0,2 \mathrm{Mol} \mathrm{LiOCN}$ gegossen, aus $0,1 \mathrm{Mol} \mathrm{Li}_{2} \mathrm{SO}_{4} \cdot \mathrm{H}_{2} \mathrm{O}+0,1 \mathrm{Mol} \mathrm{Ba}(\mathrm{OCN})_{2}$ dargestellt und durch Vakuumdestillation auf 
ein Volumen von $50 \mathrm{ml}$ konzentriert.* Die Farbe der Lösung wurde dabei etwas dunkler.

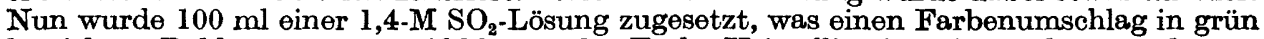
bewirkte. Bald setzte unter Abblassen der Farbe Kristallisation ein, und es wurde nun während $30 \mathrm{Min} \mathrm{SO}_{2}$ in die Lösung eingeleitet. Die Kristalle wurden wie vorher beschreiben abfiltriert und gewaschen und in Vakuum unter Lichtausschluss getrocknet und auf. bewahrt. Ausbeute 9,3 g = 88\%. Kleine Prismen von der Dichte 3,13 \pm 0.01 bei $20^{\circ}$, pyknometrisch mit Brombenzol und Xylol als Sperrflüssigkeiten bestimmt. Bei der Analyse wurden $\mathrm{Cu}$ als CuSCN und $\mathrm{N}$ nach Kjeldahl bestimmt. (Gef. Cu 60,15; $\mathrm{N} 13,29$; Ber. für CuOCN (105,58): Cu 60,23; N 13,27).

\section{Darstellung von Bariumeyanat}

$\mathrm{Zu}$ einer Lösung von $13,0 \mathrm{~g} \mathrm{NaOCN}$ in $115 \mathrm{ml} \mathrm{H}_{2} \mathrm{O}$ wurden $0,3 \mathrm{~g} \mathrm{Ba}\left(\mathrm{ClO}_{4}\right)_{2}$ (Anhydrid) gesetzt und der kleine Niederschlag von $\mathrm{BaCO}_{3}$ abfiltriert. Ferner wurden $33,6 \mathrm{~g} \mathrm{Ba}\left(\mathrm{ClO}_{4}\right)_{2}$ in $115 \mathrm{ml}$ reinem Methanol gelöst und die beiden Lösungen gemischt. Auskristallisieren setzte fast sofort ein. Nach Aufbewahren über Nacht in Kälteschrank, wurden die Kristalle abfiltriert mit Methanol und Äther gewaschen und in Vakuum getrocknet. Ausbeute 15,8 $\mathrm{g}=71 \%$. (Gef. Ba 62,35. Ber. für Ba $\left.(\mathrm{OCN})_{2}(221,38) \mathrm{Ba} 62,04\right)$. Das Salz ist auch wiederholt aus Methanollösungen von $\mathrm{Ba}(\mathrm{SCN})_{2}$ mit ungefähr derselben Ausbeute dargestellt worden.

\section{Darstellung von o-Tolylisocyanat}

$\mathrm{Zu}$ einer Lösung von $10,8 \mathrm{ml}$ konz. $\mathrm{H}_{2} \mathrm{SO}_{4}$ in $100 \mathrm{ml} \mathrm{H}_{2} \mathrm{O}$ wurden $10,7 \mathrm{ml}$ o-Toluidin gesetzt und das Gemisch bis zur vollständigen Auflösung der Base gewärmt. Die eisgekühlte Lösung wurde nun mit 7,5 $\mathrm{g} \mathrm{NaNO}_{2}$ in $10 \mathrm{ml} \mathrm{H}_{2} \mathrm{O}$ diazotiert. Farbe der Lösung blassgelb. Zusatz einer Lösung von $10 \mathrm{~g} \mathrm{KOCN}$ in $15 \mathrm{mI} \mathrm{H}_{2} \mathrm{O}$ und sofort $5 \mathrm{~g} \mathrm{KCu}_{3}(\mathrm{OCN})_{4}$. Ein braunes Addukt von Katalysator und Diazoniumsalz endstand sofort und bei dessen Zersetzung lebhafte Stickstoffentwicklung und Abscheiden einer Flüssigkeit. Sofortiges Ausschütteln mit $70 \mathrm{ml}$ eisgekühltem Chloroform, das stark braun gefärbt wurde. Das ganze wurde durch ein grosses Glasfilter G2 filtriert und die Chloroformschicht schnell absepariert. Zu der lichtgrünen Wasserlösung wurden weitere $3 \mathrm{~g}$ des Katalysators gesetzt, wobei nochmals Gasentwicklung eintrat, jedoch schwächer als vorher. Erneutes Ausschütteln mit $\mathrm{CHCl}_{3}$, das sich diesmals schwächer braun färbte. Die Prozedur wurde ein drittes Mal mit $3 \mathrm{~g}$ Katalysator wiederholt, wobei kaum Gasentwicklung stattfand und das Chloroform fast farblos blieb. Die vereinigten Chloroformextrakte wurden sofort mit $\mathrm{P}_{2} \mathrm{O}_{5}$ getrocknet und das Lösungsmittel in Vakuum bei einer Badtemperatur unter $40^{\circ}$ abdestilliert. Gewicht des flüssigen Rohprodukts von 3 solchen Synthesen $37 \mathrm{~g}$. Zunächst wurde in Vakuum fraktioniert, wobei als Mittelfraktion $16 \mathrm{~g}$ Flüssigkeit gewonnen wurden. $30 \mathrm{~g}$ derart dargestelltes Präparat lieferten bei einer zweiten Fraktio. nierung unter gewöhnlichem Druck $21 \mathrm{~g}$ zwischen $183^{\circ}$ und $187^{\circ}$ siedendes Isocyanat = $28 \%$.

Die Synthese des o-Tolylisocyanats ist auch mit Benzol als Extraktionsmittel ausgeführt worden. Die Abtrennung der Benzolschicht geht jedoch langsamer, was sich in einer verschlechterten Ausbeute kundgibt.

Wie ich gefunden habe, enthält das mit $\mathbf{C H C l}_{3}$ oder $\mathrm{CCl}_{4}$ extrahierte Tolylisocyanat immer kleine Mengen von Chlor, und dies gilt auch für das durch Katalyse nach Gattermann hergestellte. Das o-Tolylisocyanat ist wie Phenylisocyanat zu Tränen reizend, jedoch nicht so stark, dass Arbeiten damit eigentliche Unannehmlichkeiten mitführt.

$$
\begin{gathered}
o-\mathrm{Tolyl} \text { carbaminsäure-o-Tolylester aus o-Tolyl- } \\
\text { isocyanat }
\end{gathered}
$$

In $6,0 \mathrm{~g}$ wie oben beschrieben dargestelltes $\mathrm{CH}_{3} \mathrm{C}_{6} \mathrm{H}_{4} \mathrm{NCO}$ wurden 4,87 $\mathrm{g}$ o-Kresol gelöst, was unter Temperaturerniedrigung geschah. Die Lösung wurde auf dem Wasserbade gewärmt, wobei der Inhalt des Kolbens bald zu einem Kristallkuchen erstarrte.

* Eine Wasserlösung von LiOCN kann ebensowenig wie die Lösungen von Li-Halogeniden durch Destillation konzentriert werden ohne durch Hydrolyse alkalisch zu werden. Diese Tatsache wirkt aber in diesem Falle kaum belästigend, da die Lösung bei Zusatz von $\mathrm{SO}_{2}$ sauer wird.

Acta Chem. Scand. 11 (1957) No. 10 
Nach etwa 7 Stunden wurde das Reaktionsprodukt pulverisiert. Gewicht $10,70 \mathrm{~g}$ statt theor. 10,87. Die Substanz, die nicht mehr nach Isocyanat roch, wurde zwei Stunden bei Zimmertemperatur mit $50 \mathrm{ml}$ Benzin digeriert, dieses scharf abgesogen, die Kristalle mit etwas Benzin gewaschen und in Vakuum getrocknet. Gewicht 9,34 g. $2 \mathrm{~g}$ der Sub. stans wurden nun aus $5 \mathrm{ml}$ warmen Eisessig kristallisiert, wobei $1,34 \mathrm{~g}$ zurückgewonnen wurden. Schmelzp. 115-118 . Durch zwei weitere Kristallisationen aus Eisessig konnte der Schmelzpunkt auf $124-126^{\circ}$ erhöht werden aber nicht weiter. Prismen aus Eisessig. Von Gattermann wurde als Schmelzpunkt $126^{\circ}$ gefunden.

\section{LITERATUR}

1. Clarke, F. W. und Owens, M. E. Am. Chem. J. $3(1881-82) 350$.

2. Graf, R. Chem. Ber. 89 (1956) 1071.

3. Ripan, R. Bul. Soc. Stiinte Cluj. 4 (1928) 144; Chem. Zentr. 1929 I 2905.

4. Cohen, E. Z. physik. Chem. 14 (1894) 71; Gmelin Handb. d. anorg. Chem. 8. Aufl. Bd 32-33, S. 85 .

5. Dietz, R. Z. anorg. Chem. 20 (1899) 253.

6. Birckenbech, L. und Kolb, H. Ber. 68 (1935) 895.

7. Ley, H. Z. physik. Chem. 30 (1899) 247.

8. Rao, S. V. R. und Watson, H. E. J. Phys. Chem. 32 (1928) 1354.

9. Naumann, A. Ber. 37 (1904) 4335.

10. Wöhler, L. Gilberts Annalen 73166.

11. Knoll \& Co. D. R. P. 275200; Chem. Zentr. 1914 II 277.

12. Amell, A. R. J. Am. Chem. Soc. 78 (1956) 6234.

13. Wöhler, L. und Berthmann, A. Ber. 62 (1929) 2754.

14. Gattermann, L. und Cantzler, A. Ber. 23 (1890) 1225; 25 (1892) 1086.

Eingegangen am 9. Juli 1957. 